\title{
Integrability of Lie systems and some of its applications in physics
}

\author{
José F. Cariñena, Javier de Lucas and Manuel F. Rañada \\ Departamento de Física Teórica, Facultad de Ciencias, Universidad de Zaragoza, \\ 50009 Zaragoza, Spain \\ E-mail: jfc@unizar.es, dlucas@unizar.es, mfran@unizar.es
}

\begin{abstract}
The geometric theory of Lie systems will be used to establish integrability conditions for several systems of differential equations, in particular, Riccati equations and Ermakov systems. Many different integrability criteria in the literature will be analysed from this new perspective and some applications in physics will be given.
\end{abstract}

\section{Introduction: Non-autonomous systems of differential equations}

Non-autonomous systems of differential equations play a relevant rôle in many physical theories, describing evolution in terms of either time or any other parameter. For instance Hamilton equations are systems of first-order differential equations and EulerLagrange equations for regular Lagrangians are systems of second-order differential equations. These systems also appear in many other problems, and the theorem of local existence and uniqueness of solutions is of a crucial importance. However, it is not possible to finding explicit solutions of such systems and approximation methods have been developed for dealing with them. This gives even more relevance to the explicitly solvable examples which can be used to test approximation methods. In the linear case there is a linear superposition principle allowing us to write the general solution as a linear combination of a fundamental set of solutions. Particular solutions are also used to reduce the problem to a simpler one (see e.g. [1] for Riccati equations). Our aim in this paper is to analyse sufficient conditions for integrability of a particular case of non autonomous systems, the so called Lie systems [2, 3, 4, 5]. We shall understand that to find a solution means to reduce the problem to some quadratures.

We remark that a system of second-order differential equations in $n$ variables is related with a system of first-order equations in $2 n$ variables,

$$
\ddot{x}^{i}=F^{i}(x, \dot{x}, t) \Longrightarrow\left\{\begin{array}{rl}
\dot{x}^{i} & =v^{i} \\
\dot{v}^{i} & =F^{i}(x, v, t)
\end{array}, \quad i=1, \ldots n .\right.
$$

and therefore it is enough to restrict ourselves to study systems of first-order differential equations. For instance, for the harmonic oscillator with a $t$-dependent angular 
frequency $\omega(t)$ :

$$
\ddot{x}=-\omega^{2}(t) x \Longleftrightarrow\left\{\begin{array}{l}
\dot{x}=v \\
\dot{v}=-\omega^{2}(t) x
\end{array} .\right.
$$

From the geometric viewpoint, a system

$$
\dot{x}^{i}=X^{i}(x, t), \quad i=1, \ldots, n,
$$

is associated with the $t$-dependent vector field

$$
X=X^{i}(x, t) \frac{\partial}{\partial x^{i}}
$$

whose integral curves are determined by the solutions of (3). In particular, for (21),

$$
X=v \frac{\partial}{\partial x}-\omega^{2}(t) x \frac{\partial}{\partial v},
$$

The general solution of the inhomogeneous linear differential equation

$$
\frac{d x}{d t}=b_{0}(t)+b_{1}(t) x
$$

can be found with two quadratures. It is given by

$$
x(t)=\exp \left(\int_{0}^{t} b_{1}(s) d s\right) \times\left(x_{0}+\int_{0}^{t} b_{0}\left(t^{\prime}\right) \exp \left(-\int_{0}^{t^{\prime}} b_{1}(s) d s\right) d t^{\prime}\right),
$$

and when the systems we are dealing with are linear, there is a linear superposition principle allowing us to find the general solution as a linear combination of $n$ particular solutions. For instance, for (2) if we know a particular solution the general solution can be found by means of one quadrature, while if we know two particular solutions, $x_{1}$ and $x_{2}$, the general solution is a linear combination (no quadrature is needed) $x(t)=k_{1} x_{1}(t)+k_{2} x_{2}(t)$. A less known fact is the existence of other systems for which one can write the general solution as a (maybe nonlinear) function of a fundamental set of solutions and a set of constants determining each particular solution. The characterization of such systems is due to Lie [2] and these Lie systems appear quite often not only in mathematics but also in both classical and quantum physics [4, 5, 6, 17, 8, 9, 10, 11, the almost ubiquitous Riccati equation,

$$
\frac{d x(t)}{d t}=b_{2}(t) x^{2}(t)+b_{1}(t) x(t)+b_{0}(t) .
$$

which appears in the reduction process from a linear second-order equation when taking into account its dilation invariance, or in factorization methods, being one important example [1, 6, 12, 13]. For such Riccati equation, if a particular solution is known, the general solution is obtained by two quadratures; if two particular solutions are known the problem reduces to one quadrature; and finally, when three particular solutions are known, $x_{1}, x_{2}$ and $x_{3}$, the general solution can be found from the cross ratio relation 12 ]

$$
\frac{x-x_{1}}{x-x_{2}}: \frac{x_{3}-x_{1}}{x_{3}-x_{2}}=k
$$


which provides us a nonlinear superposition rule.

All these properties can be better understood in the framework of Lie systems [2], conveniently extended in some cases to include second-order differential equations systems. These systems have a lot of applications not only in mathematics but also in many different branches of physics, both at the classical and the quantum level [3], some of them determined by $t$-dependent Hamiltonian that in general are not easy to handle. Furthermore, Lie systems are also relevant in Control Theory.

The paper is organized as follows. Next section is devoted to first recall some important properties of Lie systems, and then to extend the theory for systems of secondorder differential equations. Moreover, we illustrate the theory with some important examples with applications in physics and other relevant examples as Milne-Pinney [14] equation $\ddot{x}=-\omega^{2}(t) x+k / x^{3}$, which is usually studied together with the timedependent harmonic oscillator $\ddot{y}+\omega^{2}(t) y=0$, the so-called Ermakov system. Pinney showed in a short paper [15] that the general solution of the first equation can be written as a nonlinear superposition of two solutions of the associated harmonic oscillator. A generalisation of Ermakov systems and the corresponding Ermakov invariants, which appear as first integrals in a natural way, will also be analysed. It will be shown that all these properties can be better understood in the framework of Lie systems [2], conveniently extended in some cases to include second-order differential equations systems. The perspective of such systems as Lie systems is very important because they have a lot of applications not only in mathematics but also in many different branches of physics, both at the classical and the quantum level [3]. In particular, some $t$-dependent Hamiltonian systems are of this class, and Lie systems are also relevant in Control Theory.

Section 3 is devoted to explain the reduction method which is the main ingredient in the setting of sufficient integrability conditions that are proposed in Section 4. This is based on the affine action of the group of curves in the associated Lie group on the set of Lie systems with such a group (see [16] for a geometric interpretation) and generalizes the results obtained in [1] for Riccati equation. The integrability conditions are used in Section 5 for studying Caldirola-Kanai oscillator [17, 18] and the possibility of reduction to an autonomous system is proved as a consequence of the theory. Arbitrary timedependent systems are also studied and explicit time-dependences are proved to satisfy the above mentioned integrability conditions and therefore to correspond to explicitly solvable models.

\section{Lie systems: A geometric approach}

The conditions for the system (3) to admit a superposition rule, i.e. there exists a function $\Phi: \mathbb{R}^{n(m+1)} \rightarrow \mathbb{R}^{n}, x=\Phi\left(x_{(1)}, \ldots, x_{(m)} ; k_{1}, \ldots, k_{n}\right)$, such that its general solution can be written as $x(t)=\Phi\left(x_{(1)}(t), \ldots, x_{(m)}(t) ; k_{1}, \ldots, k_{n}\right)$, where $\left\{x_{(a)}(t) \mid a=\right.$ $1, \ldots, m\}$ is a fundamental set of particular solutions and $k=\left(k_{1}, \ldots, k_{n}\right)$ is a set of $n$ arbitrary constants, were studied by Lie [2]. Under very general conditions [5] the 
necessary and sufficient conditions is that the associated $t$-dependent vector field $X(x, t)$ can be written as a linear combination

$$
X(x, t)=\sum_{\alpha=1}^{r} b_{\alpha}(t) X_{(\alpha)}(x),
$$

such that the vector fields $\left\{X_{(\alpha)} \mid \alpha=1, \ldots, r\right\}$ generate a finite-dimensional real Lie algebra, i.e. there exist $r^{3}$ real numbers $c_{\alpha \beta}{ }^{\gamma}$, such that

$$
\left[X_{(\alpha)}, X_{(\beta)}\right]=\sum_{\gamma=1}^{r} c_{\alpha \beta}^{\gamma} X_{(\gamma)}, \quad \alpha, \beta=1, \ldots, r .
$$

Linear systems are particular examples whose associated Lie algebra is $\mathfrak{g l}(n, \mathbb{R})$ and $m=n$ in the homogeneous case, or the corresponding affine algebra and $m=n+1$ in the inhomogeneous one. Riccati equation is another example for which $X(x, t)=$ $b_{0}(t) Y_{0}(x)+b_{1}(t) Y_{1}(x)+b_{2}(t) Y_{2}(x)$ with

$$
Y_{0}(x)=\frac{\partial}{\partial x}, \quad Y_{1}(x)=x \frac{\partial}{\partial x}, \quad Y_{2}(x)=x^{2} \frac{\partial}{\partial x} .
$$

with commutation relations

$$
\left[Y_{0}, Y_{1}\right]=Y_{0}, \quad\left[Y_{2}, Y_{1}\right]=-Y_{2}, \quad\left[Y_{2}, Y_{0}\right]=-2 Y_{1}
$$

closing on a $\mathfrak{s l}(2, \mathbb{R})$ Lie algebra [1, 13]. There is an action $\Phi_{\text {Ric }}: S L(2, \mathbb{R}) \times \overline{\mathbb{R}} \rightarrow \overline{\mathbb{R}}$ of the Lie group $S L(2, \mathbb{R})$ on $\overline{\mathbb{R}} \equiv \mathbb{R} \cup \infty$ given by

$$
\begin{aligned}
\Phi_{\text {Ric }}(A, x) & =\frac{\alpha x+\beta}{\gamma x+\delta}, \quad \text { if } \quad x \neq-\frac{\delta}{\gamma}, \\
\Phi_{\text {Ric }}(A, \infty) & =\alpha / \gamma, \quad \Phi_{\text {Ric }}(A,-\delta / \gamma)=\infty,
\end{aligned}
$$

where the matrix $A \in S L(2, \mathbb{R})$ is

$$
A=\left(\begin{array}{ll}
\alpha & \beta \\
\gamma & \delta
\end{array}\right)
$$

such that the fundamental vector fields of this action are the linear combinations of the vector fields $Y_{0}, Y_{1}$ and $Y_{2}$.

Another very relevant example of Lie system is given by a $t$-dependent rightinvariant vector field in a Lie group $G$. A right-invariant vector field $X^{\mathrm{R}}$ is one such that $X^{\mathrm{R}}(g)=R_{g * e} X^{\mathrm{R}}(e)$. If $\left\{\mathrm{a}_{1}, \ldots, \mathrm{a}_{r}\right\}$ is a basis of $T_{e} G$ and define the right-invariant vector fields $X_{\alpha}^{\mathrm{R}}$ by $X_{\alpha}^{\mathrm{R}}(g)=R_{g * e} \mathrm{a}_{\alpha}$, then the $t$-dependent right-invariant vector field $\bar{X}(g, t)=-\sum_{\alpha=1}^{r} b_{\alpha}(t) X_{\alpha}^{\mathrm{R}}(g)$ defines a Lie system in $G$ whose integral curves are solutions of the system $\dot{g}=-\sum_{\alpha=1}^{r} b_{\alpha}(t) X_{\alpha}^{\mathrm{R}}(g)$, and when applying $R_{g^{-1}}$ to both sides we see that $g(t)$ satisfies

$$
R_{g^{-1}(t) * g(t)} \dot{g}(t)=-\sum_{\alpha=1}^{r} b_{\alpha}(t) \mathrm{a}_{\alpha} \in T_{e} G .
$$

Right-invariance means that it is enough to know one solution, for instance the one starting from the neutral element $g(t)$, to know all the solutions of the equation with 
any initial condition, i.e. we obtain the solution $g^{\prime}(t)$ with initial condition $g^{\prime}(0)=g_{0}$ as $R_{g(0)} g(t)$. A generalization of the method by Wei-Norman [19] is very useful in solving such equation, and furthermore reduction techniques can also be used [20]. Finally, as right-invariant vector fields $X^{\mathrm{R}}$ project on the fundamental vector fields in each homogeneous space for $G$, the solution of (9) allows us to find the general solution for the corresponding Lie system in each homogeneous space. Conversely, the knowledge of particular solutions of the associated system in a homogeneous space gives us a method for reduction the problem to the corresponding isotopy group [20]. This equation is also important because any Lie system described by a $t$-dependent vector field in a manifold $M$ like (6) where the vector fields are complete and close the same commutation relations as the basis $\left\{\mathrm{a}_{1}, \ldots, \mathrm{a}_{r}\right\}$ determines an action $\Phi_{\mathrm{LieG}}: G \times M \rightarrow M$ such that the vector field $X_{\alpha}$ is the fundamental vector field corresponding to $\mathrm{a}_{\alpha}$, and moreover, the integral curves of the t-dependent vector field are obtained from the solutions of thr equation (9). More explicitly, the general solution of the given Lie system are $x(t)=\Phi_{\text {LieG }}\left(g(t), x_{0}\right)$, where $x_{0}$ is an initial condition of the solution and $g(t)$ is the solution for (9) with $g(0)=e$.

The search for the number $m$ of solutions and the superposition function $\Phi$ have recently been studied from a geometric perspective [5]. Essentially we should consider 'diagonal prolongations' to $\mathbb{R}^{n(m+1)}, \widetilde{X}\left(x_{(0)}, \ldots, x_{(m)}, t\right)$, of the $t$-dependent vector field $X(x, t)=X^{i}(t, x) \partial / \partial x^{i}$, given by

$$
\widetilde{X}\left(x_{(0)}, \ldots, x_{(m)}, t\right)=\sum_{a=0}^{m} X_{a}\left(x_{(a)}, t\right), \quad t \in \mathbb{R},
$$

where $X_{a}\left(x_{(a)}, t\right)=X^{i}\left(x_{(a)}, t\right) \partial / \partial x_{(a)}^{i}$, such that the extended system admits $n$ independent integrals of motion, which define in an implicit way the superposition function.

As another example of Lie system of physical relevance, consider the $t$-dependent harmonic oscillator described by a Hamiltonian

$$
H(x, p, t)=\frac{1}{2} \frac{p^{2}}{m(t)}+\frac{1}{2} m(t) \omega^{2}(t) x^{2},
$$

whose Hamilton equations are given by

$$
\left\{\begin{array}{l}
\dot{x}=\frac{\partial H}{\partial p}=\frac{p}{m(t)} \\
\dot{p}=-\frac{\partial H}{\partial x}=-m(t) \omega^{2}(t) x .
\end{array}\right.
$$

Its solutions are the integral curves of the $t$-dependent vector field

$$
X(x, p, t)=\frac{1}{m(t)} p \frac{\partial}{\partial x}-m(t) \omega^{2}(t) x \frac{\partial}{\partial p} .
$$

If we consider the set of vector fields

$$
X_{0}=p \frac{\partial}{\partial x}, \quad X_{1}=\frac{1}{2}\left(x \frac{\partial}{\partial x}-p \frac{\partial}{\partial p}\right), \quad X_{2}=-x \frac{\partial}{\partial p},
$$


which close on a $\mathfrak{s l}(2, \mathbb{R})$ Lie algebra with commutation relations

$$
\left[X_{0}, X_{1}\right]=X_{0}, \quad\left[X_{2}, X_{1}\right]=-X_{2}, \quad\left[X_{2}, X_{0}\right]=-2 X_{1},
$$

then the $t$-dependent vector field (10) can be written as a linear combination with $t$ dependent coefficients $X(\cdot, t)=\sum_{\alpha=0}^{2} b_{\alpha}(t) X_{\alpha}(\cdot)$, with

$$
b_{0}(t)=\frac{1}{m(t)}, \quad b_{1}(t)=0, \quad b_{2}(t)=m(t) \omega^{2}(t)
$$

Remark that the vector fields of this example and those of Riccati equation close the same commutation relations.

As an instance of t-dependent harmonic oscillator we next develop the one with damping term, with equation of motion

$$
\frac{d}{d t}\left(m_{0} \dot{x}\right)+m_{0} \mu \dot{x}+k x=0, \quad k=m_{0} \omega^{2},
$$

admits a Hamiltonian description with a t-dependent Hamiltonian [17, 18]

$$
H=\frac{p^{2}}{2 m_{0}} \exp (-\mu t)+\frac{1}{2} m_{0} \exp (\mu t) \omega^{2} x^{2}=\frac{p^{2}}{2 m(t)}+\frac{1}{2} m(t) \omega^{2} x^{2}
$$

with $m(t)=m_{0} \exp (\mu t)$. The time-dependent coefficients as a Lie system are:

$$
b_{0}(t)=\frac{\exp (-\mu t)}{m_{0}}, \quad b_{1}(t)=0, \quad b_{2}(t)=m_{0} \exp (\mu t) \omega^{2} .
$$

We can also consider the time-dependent frequency harmonic oscillator

$$
H=\frac{1}{2} p^{2}+\frac{1}{2} F(t) \omega_{0}^{2} x^{2},
$$

whose associated $t$-dependent vector field $X$ is a linear combination $X(\cdot, t)=$ $F(t) \omega_{0}^{2} X_{2}(\cdot)+X_{0}(\cdot)$ with coefficients:

$$
b_{0}(t)=1, \quad b_{1}(t)=0, \quad b_{2}(t)=F(t) \omega_{0}^{2} .
$$

The theory of Lie systems can be extended to include systems of second-order differential equations. Such a system whose associated system of first-order differential equations is a Lie system is called SODE Lie system [21]. This is the case of the 1dimensional harmonic oscillator (2), where $X(x, v, t)$ is a linear combination $X(\cdot, t)=$ $X_{2}(\cdot)-\omega^{2}(t) X_{1}(\cdot)$ of the vector fields $X_{1}(x, v)=x \partial / \partial v$ and $X_{2}(x, v)=v \partial / \partial x$ that close a finite-dimensional Lie algebra with $X_{3}(x, v)=1 / 2(x \partial / \partial x-v \partial / \partial v)$. Similarly for the isotropic 2-dimensional case,

$$
\left\{\begin{array}{l}
\ddot{x}_{1}=-\omega^{2}(t) x_{1} \\
\ddot{x}_{2}=-\omega^{2}(t) x_{2}
\end{array}\right.
$$

but with $X_{1}\left(x_{1}, x_{2}, v_{1}, v_{2}\right)=x_{1} \partial / \partial v_{1}+x_{2} \partial / \partial v_{2}, X_{2}\left(x_{1}, x_{2}, v_{1}, v_{2}\right)=v_{1} \partial / \partial x_{1}+v_{2} \partial / \partial x_{2}$ and $X_{3}\left(x_{1}, x_{2}, v_{1}, v_{2}\right)=1 / 2\left(x_{1} \partial / \partial x_{1}+x_{2} \partial / \partial x_{2}-v_{1} \partial / \partial v_{1}-v_{2} \partial / \partial v_{2}\right)$, in both cases with associated Lie algebra $\mathfrak{s l}(2, \mathbb{R})$, 
The general theory of Lie systems can now be used and so we can obtain a first integral, $F\left(x_{1}, x_{2}, v_{1}, v_{2}\right)=x_{1} v_{2}-x_{2} v_{1}$, which is nothing but the Wronskian of two solutions, or the angular momentum from the physical viewpoint. This may be seen as a partial superposition rule: if $x_{1}(t)$ is a solution of the first equation, then we obtain for each real number $k$ the first-order differential equation for the variable $x_{2}$, $x_{1}(t) d x_{2} / d t=k+\dot{x}_{1}(t) x_{2}$, from where $x_{2}$ can be found to be given by

$$
x_{2}(t)=k^{\prime} x_{1}(t)+k x_{1}(t) \int^{t} \frac{d \zeta}{x_{1}^{2}(\zeta)} .
$$

In a similar way, with three copies of the same harmonic oscillator, one obtains two first integrals, $F_{1}\left(x_{1}, x_{2}, x, v_{1}, v_{2}, v\right)=x v_{1}-x_{1} v$ and $F_{2}\left(x_{1}, x_{2}, x, v_{1}, v_{2}, v\right)=x v_{2}-x_{2} v$ that produce a superposition rule, because from

$$
\left\{\begin{array}{l}
x v_{2}-x_{2} v=k_{1} \\
x_{1} v-v_{1} x=k_{2}
\end{array}\right.
$$

we obtain the expected superposition rule [21]:

$$
x=k_{1} x_{1}+k_{2} x_{2}, \quad v=k_{1} v_{1}+k_{2} v_{2} .
$$

It has only recently been pointed out [21] that the differential equation [14, 15]

$$
\ddot{x}=-\omega^{2}(t) x+\frac{k}{x^{3}},
$$

is also a Lie system with associated Lie algebra $\mathfrak{s l}(2, \mathbb{R})$ : the corresponding $t$-dependent vector field is

$$
X(x, v, t)=v \frac{\partial}{\partial x}+\left(-\omega^{2}(t) x+\frac{k}{x^{3}}\right) \frac{\partial}{\partial v} .
$$

which is a linear combination $X=L_{2}-\omega^{2}(t) L_{1}$ with

$$
L_{1}=x \frac{\partial}{\partial v}, \quad L_{2}=\frac{k}{x^{3}} \frac{\partial}{\partial v}+v \frac{\partial}{\partial x}
$$

which are such that

$$
\left[L_{1}, L_{2}\right]=2 L_{3}, \quad\left[L_{3}, L_{1}\right]=L_{1}, \quad\left[L_{3}, L_{2}\right]=-L_{2}
$$

with

$$
L_{3}=\frac{1}{2}\left(x \frac{\partial}{\partial x}-v \frac{\partial}{\partial v}\right) .
$$

Another important example of Lie system with the same Lie algebra $\mathfrak{s l}(2, \mathbb{R})$ is the Ermakov system:

$$
\left\{\begin{aligned}
\dot{x} & =v_{x} \\
\dot{v}_{x} & =-\omega^{2}(t) x \\
\dot{y} & =v_{y} \\
\dot{v}_{y} & =-\omega^{2}(t) y+\frac{1}{y^{3}}
\end{aligned}\right.
$$


with associated $t$-dependent vector field

$$
X=v_{x} \frac{\partial}{\partial x}+v_{y} \frac{\partial}{\partial y}-\omega^{2}(t) x \frac{\partial}{\partial v_{x}}+\left(-\omega^{2}(t) y+\frac{1}{y^{3}}\right) \frac{\partial}{\partial v_{y}},
$$

which is a linear combination with $t$-dependent coefficients, $X=-\omega^{2}(t) X_{1}+X_{2}$, with

$$
X_{1}=x \frac{\partial}{\partial v_{x}}+y \frac{\partial}{\partial v_{y}}, \quad X_{2}=v_{x} \frac{\partial}{\partial x}+v_{y} \frac{\partial}{\partial y}+\frac{1}{y^{3}} \frac{\partial}{\partial v_{y}}
$$

This system is made up by two Lie systems closing on a $\mathfrak{s l}(2, \mathbb{R})$ algebra: the first one is a harmonic oscillator and the second one is a Pinney equation. The system admits a first integral of the motion which can be found as a solution of $X_{1} F=X_{2} F=0$ because these two conditions imply that $X_{3} F=1 / 2\left[X_{1}, X_{2}\right] F=0$. But $X_{1} F=0$ means that $F\left(x, y, v_{x}, v_{y}\right)=\bar{F}(x, y, \xi)$ with $\xi=x v_{y}-y v_{x}$, and then $X_{2} F=0$ is written

$$
v_{x} \frac{\partial \bar{F}}{\partial x}+v_{y} \frac{\partial \bar{F}}{\partial y}+\frac{x}{y^{3}} \frac{\partial \bar{F}}{\partial \xi}=0
$$

and from the associated characteristics system we obtain

$$
\frac{x d y-y d x}{\xi}=\frac{y^{3} d \xi}{x} \Longrightarrow \frac{d(x / y)}{\xi}+\frac{y d \xi}{x}=0
$$

the following first integral is found:

$$
\psi\left(x, y, v_{x}, v_{y}\right)=\left(\frac{x}{y}\right)^{2}+\xi^{2}=\left(\frac{x}{y}\right)^{2}+\left(x v_{y}-y v_{x}\right)^{2}
$$

which is the well-known Ermakov invariant. A possible generalisation of the Ermakov system (16) is given by:

$$
\left\{\begin{array}{l}
\ddot{x}=\frac{1}{x^{3}} f(y / x)-\omega^{2}(t) x \\
\ddot{y}=\frac{1}{y^{3}} g(y / x)-\omega^{2}(t) y
\end{array},\right.
$$

which reduces to (16) for $f(u)=0$ and $g(u)=1$.

This system can be written as a first-order one by doubling the number of degrees of freedom by introducing the new variables $v_{x}$ and $v_{y}$ :

$$
\left\{\begin{aligned}
\dot{x} & =v_{x} \\
\dot{v}_{x} & =-\omega^{2}(t) x+\frac{1}{x^{3}} f(y / x) \\
\dot{y} & =v_{y} \\
\dot{v}_{y} & =-\omega^{2}(t) y+\frac{1}{y^{3}} g(y / x)
\end{aligned}\right.
$$

which determines the integral curves of the vector field

$$
X=v_{x} \frac{\partial}{\partial x}+v_{y} \frac{\partial}{\partial v_{y}}+\left(-\omega^{2}(t) x+\frac{1}{x^{3}} f(y / x)\right) \frac{\partial}{\partial v_{x}}+\left(-\omega^{2}(t) y+\frac{1}{y^{3}} g(y / x)\right) \frac{\partial}{\partial v_{y}} .
$$


Such vector field can be written as a linear combination

$$
X=N_{2}-\omega^{2}(t) N_{1}
$$

where $N_{1}$ and $N_{2}$ are the vector fields

$$
N_{1}=x \frac{\partial}{\partial v_{x}}+y \frac{\partial}{\partial v_{y}}, \quad N_{2}=v_{x} \frac{\partial}{\partial x}+\frac{1}{x^{3}} f(y / x) \frac{\partial}{\partial v_{x}}+v_{y} \frac{\partial}{\partial y}+\frac{1}{y^{3}} g(y / x) \frac{\partial}{\partial v_{y}},
$$

Note that these vector fields generate a three-dimensional real Lie algebra with a third generator

$$
N_{3}=\frac{1}{2}\left(x \frac{\partial}{\partial x}-v_{x} \frac{\partial}{\partial v_{x}}+y \frac{\partial}{\partial y}-v_{y} \frac{\partial}{\partial v_{y}}\right)
$$

In fact, as

$$
\left[N_{1}, N_{2}\right]=2 N_{3}, \quad\left[N_{3}, N_{1}\right]=N_{1}, \quad\left[N_{3}, N_{2}\right]=-N_{2}
$$

they generate a Lie algebra isomorphic to $\mathfrak{s l}(2, \mathbb{R})$. Therefore the system is a Lie system.

There exists a first integral for the motion, $F: \mathbb{R}^{4} \rightarrow \mathbb{R}$, for any $\omega^{2}(t)$, which can be found in a similar way and we will arrive to the first integral:

$$
\frac{1}{2}\left(x v_{y}-y v_{x}\right)^{2}+\int^{x / y}\left[-\frac{1}{u^{3}} f\left(\frac{1}{u}\right)+u g\left(\frac{1}{u}\right)\right] d u .
$$

This first integral allows us to determine a solution of one subsystem in terms of a solution of the other equation.

Finally, we can revisit Pinney equation by considering a system made up by a Pinney equation in the $x$ variable with two associated harmonic oscillator equations for variables $y$ and $z$. by considering the system of first-order differential equations:

$$
\left\{\begin{aligned}
\dot{x} & =v_{x} \\
\dot{y} & =v_{y} \\
\dot{z} & =v_{z} \\
\dot{v}_{x} & =-\omega^{2}(t) x+\frac{k}{x^{3}} \\
\dot{v}_{y} & =-\omega^{2}(t) y \\
\dot{v}_{z} & =-\omega^{2}(t) z
\end{aligned}\right.
$$

which corresponds to the vector field

$$
X=v_{x} \frac{\partial}{\partial x}+v_{y} \frac{\partial}{\partial y}+v_{z} \frac{\partial}{\partial z}+\frac{k}{x^{3}} \frac{\partial}{\partial v_{x}}-\omega^{2}(t)\left(x \frac{\partial}{\partial v_{x}}+y \frac{\partial}{\partial v_{y}}+z \frac{\partial}{\partial v_{z}}\right)
$$

The vector field $X$ can be expressed as $X=N_{2}-\omega^{2}(t) N_{1}$ where the vector fields $N_{1}$ and $N_{2}$ are:

$$
N_{1}=x \frac{\partial}{\partial v_{x}}+y \frac{\partial}{\partial v_{y}}+z \frac{\partial}{\partial v_{z}}, \quad N_{2}=v_{x} \frac{\partial}{\partial x}+\frac{1}{x^{3}} \frac{\partial}{\partial v_{x}}+v_{y} \frac{\partial}{\partial y}+v_{z} \frac{\partial}{\partial z}
$$


These vector fields generate a 3-dimensional real Lie algebra with $N_{3}$ given by

$$
N_{3}=\frac{1}{2}\left(x \frac{\partial}{\partial x}-v_{x} \frac{\partial}{\partial v_{x}}+y \frac{\partial}{\partial y}-v_{y} \frac{\partial}{\partial v_{y}}+z \frac{\partial}{\partial z}-v_{z} \frac{\partial}{\partial v_{z}}\right) .
$$

In fact, as

$$
\left[N_{1}, N_{2}\right]=2 N_{3}, \quad\left[N_{3}, N_{1}\right]=N_{1}, \quad\left[N_{3}, N_{2}\right]=-N_{2}
$$

they generate a Lie algebra isomorphic to $\mathfrak{s l}(2, \mathbb{R})$. Thus this is a Lie system.

There exist three first-integrals for the distribution generated by these fundamental vector fields: the Ermakov invariant $I_{1}$ of the subsystem involving variables $x$ and $y$, the Ermakov invariant $I_{2}$ of the subsystem involving variables $x$ and $z$, and finally, the Wronskian $W$ of the subsystem involving variables $y$ and $z$. They are given by $W=y v_{z}-z v_{y}$,

$$
I_{1}=\frac{1}{2}\left(\left(y v_{x}-x v_{y}\right)^{2}+k\left(\frac{y}{x}\right)^{2}\right), \quad I_{2}=\frac{1}{2}\left(\left(x v_{z}-z v_{x}\right)^{2}+k\left(\frac{z}{x}\right)^{2}\right) .
$$

In terms of these three integrals we can obtain an explicit expression of $x$ in terms of $y, z$ and the integrals $I_{1}, I_{2}, W$ :

$$
x=\frac{\sqrt{2}}{W}\left(I_{2} y^{2}+I_{1} z^{2} \pm \sqrt{4 I_{1} I_{2}-k W^{2}} y z\right)^{1 / 2} .
$$

We can recover in this way the result of [15]: the general solution of Pinney equation can be expressed in terms of two solution of the corresponding harmonic oscillator problem with time-dependent frequency

\section{The reduction method}

Given an equation (9) on a Lie group, it may happen that the only non-vanishing coefficients are those corresponding to a subalgebra $\mathfrak{h}$ of $\mathfrak{g}$ and then the equation reduces to a simpler equation on a subgroup, involving less coordinates. An important result is that if we know a particular solution of the problem associated in a homogeneous space, the original solution reduces to one on the isotopy subgroup [20].

One can show that there is an action of the group $\mathcal{G}$ of curves in $G$ on the set of right-invariant Lie systems in $G$ (see e.g. [16] for a geometric justification), and we can take advantage of such an action for transforming a given Lie system into another simpler one.

So, if $g(t)$ is a solution of the given Lie system satisfying (9) and we choose a curve $g^{\prime}(t)$ in the group $G$, and define a curve $\bar{g}(t)$ by $\bar{g}(t)=g^{\prime}(t) g(t)$, then the new curve in $G, \bar{g}(t)$, determines a new Lie system. Indeed,

$$
R_{\bar{g}(t)^{-1} * \bar{g}(t)}(\dot{\bar{g}}(t))=R_{g^{\prime-1}(t) * g^{\prime}(t)}\left(\dot{g}^{\prime}(t)\right)-\sum_{\alpha=1}^{r} b_{\alpha}(t) \operatorname{Ad}\left(g^{\prime}(t)\right) \mathrm{a}_{\alpha},
$$


which is similar to the original one, with a different right-hand side. Therefore, the aim is to choose the curve $g^{\prime}(t)$ in such a way that the new equation be simpler. For instance, we can choose a subgroup $H$ and look for a choice of $g^{\prime}(t)$ such that the right hand side lies in $T_{e} H$, and hence if $\bar{g}(0)=e$ then $\bar{g}(t) \in H$ for all $t$. This can be done when we know a solution of the associated Lie system in $G / H$, what allows us to reduce the problem to one in the subgroup $H$, see [20].

Theorem 1 Each solution of (9) on the group $G$ can be written in the form $g(t)=$ $g_{1}(t) h(t)$, where $g_{1}(t)$ is a curve on $G$ projecting onto a solution $\tilde{g}_{1}(t)$ for the left action $\lambda$ of $G$ on the homogeneous space $G / H$ and $h(t)$ is a solution of an equation but for the subgroup $H$, given explicitly by

$$
\left(R_{h^{-1} * h} \dot{h}\right)(t)=-\operatorname{Ad}\left(g_{1}^{-1}(t)\right)\left(\sum_{\alpha=1}^{r} b_{\alpha}(t) \mathrm{a}_{\alpha}+\left(R_{g_{1}^{-1} * g_{1}} \dot{g}_{1}\right)(t)\right) \in T_{e} H .
$$

This fact is very important because one can show that Lie systems associated with solvable Lie algebras are solvable by quadratures and therefore, given a Lie system with an arbitrary $G$ having a solvable subgroup, we should look for a possible transformation from the original system to one which reduces to the subalgebra and therefore integrable by quadratures.

The result of the preceding Theorem proves that for a solvable Lie subgroup $H$ of $G$ there always exists a curve in $G$ that transforms the initial Lie system into a new one in subgroup $H$ of $G$. Nevertheless, it can be difficult to find out a solution of the equation in $G / H$ that determines this transformation. Then, to be able to obtain one is more interesting to suppose also that this transformation is a curve in a certain subset of $G$, i.e. a one-dimensional Lie subgroup. When such a transformation exists, it is easier to obtain it, but it may be that such a transformation does not exist, and the conditions

for the existence of such a transformation of a certain form are integrability conditions for the system.

We could choose for showing this assertion a particular example: Riccati equation. One can find in the literature a lot of integrability criteria for Riccati equation, all of them particular examples of the above method.

We can also consider more relevant examples in Physics, for instance, timedependent harmonic oscillators.

The results obtained for one system are valid for the other; they are essentially conditions for the equation in the group, and both are examples of Lie systems associated with the same Lie group: $S L(2, \mathbb{R})$.

\section{Integrability criteria for Lie systems}

Consider the particular case of Lie systems with associated Lie group $S L(2, \mathbb{R})$, therefore valid for Riccati equation (5), Milne-Pinney equation (14) and the time-dependent harmonic oscillator described by (10). The group $S L(2, \mathbb{R})$ contains the affine group 
(either the one generated by $X_{0}$ and $X_{1}$ or the one generated by $X_{1}$ and $X_{2}$ ), which is solvable. Therefore, a transformation from the given equation to one of this subgroup allows us to express the general solution in terms of quadratures. This happens when we know a particular solution $x_{1}$ of the given equation: $x=x_{1}+z$, what corresponds to choose

$$
\bar{g}(t)=\left(\begin{array}{cc}
1 & -x_{1} \\
0 & 1
\end{array}\right)
$$

reduces the equation to

$$
\frac{d z}{d t}=\left(2 a_{2} x_{1}+a_{1}\right) z+a_{2} z^{2}
$$

The reduction by the knowledge of two or three quadratures has also been studied from this perspective and similarly Strelchenya criterion [1, 22].

Each Riccati equation can be considered as a curve in $\mathbb{R}^{3}$ and we can transform every function in $\mathbb{R}, x(t)$, under an element of the group $\mathcal{G}$ of smooth $S L(2, \mathbb{R})$-valued curves $\operatorname{Map}(\mathbb{R}, S L(2, \mathbb{R}))$, as follows:

$$
\begin{aligned}
\Theta(A, x(t))= & \frac{\alpha(t) x(t)+\beta(t)}{\gamma(t) x(t)+\delta(t)}, \quad \text { if } x(t) \neq-\frac{\delta(t)}{\gamma(t)} \\
\Theta(A, \infty)= & \alpha(t) / \gamma(t), \quad \Theta(A,-\delta(t) / \gamma(t))=\infty \\
& \text { when } A=\left(\begin{array}{cc}
\alpha(t) & \beta(t) \\
\gamma(t) & \delta(t)
\end{array}\right) \in \mathcal{G} .
\end{aligned}
$$

The image $x^{\prime}(t)=\Theta(\bar{A}(t), x(t))$ of a curve $x(t)$ solution of the given Riccati equation satisfies a new Riccati equation with coefficients $b_{2}^{\prime}, b_{1}^{\prime}, b_{0}^{\prime}$, given by

$$
\begin{aligned}
b_{2}^{\prime} & =\bar{\delta}^{2} b_{2}-\bar{\delta} \bar{\gamma} b_{1}+\bar{\gamma}^{2} b_{0}+\bar{\gamma} \dot{\bar{\delta}}-\bar{\delta} \dot{\bar{\gamma}} \\
b_{1}^{\prime} & =-2 \bar{\beta} \bar{\delta} b_{2}+(\bar{\alpha} \bar{\delta}+\bar{\beta} \bar{\gamma}) b_{1}-2 \bar{\alpha} \bar{\gamma} b_{0}+\bar{\delta} \dot{\bar{\alpha}}-\bar{\alpha} \dot{\bar{\delta}}+\bar{\beta} \dot{\bar{\gamma}}-\bar{\gamma} \dot{\bar{\beta}} \\
b_{0}^{\prime} & =\bar{\beta}^{2} b_{2}-\bar{\alpha} \bar{\beta} b_{1}+\bar{\alpha}^{2} b_{0}+\overline{\bar{\alpha}}-\bar{\beta} \dot{\bar{\alpha}} .
\end{aligned}
$$

This expression defines an affine action of the group $\mathcal{G}$ on the set of Riccati equations. As indicated above the vector fields defining the TDHO and the Riccati equation close the same conmutation relations and both examples are related with exactly the same equation in $S L(2, \mathbb{R})$, but they correspond to different actions.

Lie systems in $S L(2, \mathbb{R})$ defined by a constant curve, a $(t)=\sum_{\alpha=0}^{2} c_{\alpha} \mathrm{a}_{\alpha}$, are integrable and the same happens for curves of the form $\mathrm{a}(t)=D(t)\left(\sum_{\alpha=0}^{2} c_{\alpha} \mathrm{a}_{\alpha}\right)$, where $D$ is an arbitrary function, because a time parametrisation reduces the problem to the previous one.

The system is essentially a Lie system on a one-dimensional Lie group.

A straightforward application of the reduction method leads to the following result which is valid not only for Riccati equation but also for any other Lie system with Lie group $S L(2, \mathbb{R})$ in which the $t$-dependent vector field be described in terms of a set of 
vector fields closing on the same commutation relations as those of Riccati equation. In this way, all these systems with the same $t$-dependent coefficients are related with the same equation in $S L(2, \mathbb{R})$ and this allows us to generalise for all these systems the integrability condition obtained in the next theorem. The other results can be generalised by means of changing the action of the Riccati equation for the action of the new Lie system.

Theorem 2 The necessary and sufficient conditions for the existence of a transformation:

$$
y^{\prime}=G(t) y \quad \text { with } \quad G(t) \geq 0
$$

generated by the transformation

$$
y^{\prime}=\Phi_{\text {Ric }}(\bar{A}(t), y) \quad \text { with } \quad \bar{A}(t)=\left(\begin{array}{cc}
\alpha(t) & 0 \\
0 & \alpha^{-1}(t)
\end{array}\right)
$$

and relating the Riccati equation in an interval $I \in \mathbb{R}$

$$
\frac{d y}{d t}=b_{0}(t)+b_{1}(t) y+b_{2}(t) y^{2}, \quad \forall t \in I, \quad\left(b_{0} b_{2}\right)(t) \neq 0,
$$

with an integrable one given by

$$
\frac{d y^{\prime}}{d t}=D(t)\left(c_{0}+c_{1} y^{\prime}+c_{2} y^{\prime 2}\right)
$$

where $c_{i}$ are real numbers, $c_{i} \in \mathbb{R}$, is that for such constants $c_{0} c_{2} \neq 0$ and

$$
D^{2}(t) c_{0} c_{2}=b_{0}(t) b_{2}(t), \quad \frac{b_{1}(t)+\frac{1}{2}\left(\frac{\dot{b}_{2}(t)}{b_{2}(t)}-\frac{\dot{b}_{0}(t)}{b_{0}(t)}\right)}{D(t)}=c_{1} .
$$

The transformation is then uniquely defined by:

$$
y^{\prime}=\sqrt{\frac{b_{2}(t) c_{0}}{b_{0}(t) c_{2}}} y .
$$

As a consequence of this theorem, given the above Riccati equation if there are constants $K$ and $L$ such that

$$
\left(b_{1}(t)+\frac{1}{2}\left(\frac{\dot{b}_{2}(t)}{b_{2}(t)}-\frac{\dot{b}_{0}(t)}{b_{0}(t)}\right)\right) \sqrt{\frac{L}{b_{0}(t) b_{2}(t)}}=K,
$$

then there exists a time-dependent linear change of variables transforming the given equation into the solvable Riccati equation:

$$
\frac{d y^{\prime}}{d t}=D(t)\left(c_{0}+c_{1} y^{\prime}+c_{2} y^{\prime 2}\right)
$$

where $c_{1}=K, c_{0} c_{2}=L$, and $D(t)$ is given as above. The existence of such constants $K$ and $L$ can be considered a sufficient condition for integrability of the given Riccati equation. Indeed, $L$ is just used to make the square to exists and can always be obtained, the real integrability condition is the existence of the constant $K$. 


\section{Some applications in physics}

\subsection{The Caldirola-Kanai oscillator}

Coming back to the time-dependent harmonic oscillator, we see that the Caldirola-Kanai model is an example of application of the previous Theorem.

We have seen that it is a Lie system with group $S L(2, \mathbb{R})$ and associated coefficients

$$
b_{0}(t)=\frac{1}{m_{0}} \exp (-\mu t), \quad b_{1}(t)=0, \quad b_{2}(t)=m_{0} \omega_{0}^{2} \exp (\mu t)
$$

Therefore, as $b_{0}(t) b_{2}(t)=\omega_{0}^{2}, b_{1}=0$ and $\left(\dot{b}_{2} / b_{2}-\dot{b}_{0} / b_{0}=2 \mu\right.$, we see that the integrability condition of the preceding theorem holds with $K=\mu / \omega_{0}$ and $L=1$, and the function $D$ is then a constant $D=\omega_{0}$. Therefore we choose $c_{0}=1, c_{1}=\mu / \omega_{0}$ and $c_{2}=1$ and this example reduces to the system

$$
\frac{d}{d t}\left(\begin{array}{l}
x \\
p
\end{array}\right)=\left(\begin{array}{cc}
\mu & \omega_{0} \\
-\omega_{0} & -\mu
\end{array}\right)\left(\begin{array}{l}
x \\
p
\end{array}\right)
$$

which can be easily integrated. This shows once again that the Caldirola-Kanai model can be reduced to an autonomous system, as a particular case of a more general situation.

\subsection{The t-dependent frequency harmonic oscillator}

In the case of the $t$-dependent frequency harmonic oscillator for which $\omega^{2}(t)=F(t) \omega_{0}$, $X(\cdot, t)=F(t) \omega_{0}^{2} X_{2}(\cdot)+X_{0}(\cdot)$, i.e. $b_{0}(t)=1, b_{1}(t)=0$ and $b_{2}(t)=F(t) \omega_{0}^{2}$, the condition on $F$ to satisfy the compatibility condition is

$$
\frac{1}{2} \frac{\dot{F}}{F}=K \omega_{0} \sqrt{F}
$$

and therefore $F$ must be of the form

$$
F(t)=\frac{1}{\left(-K \omega_{0} t+K^{\prime}\right)^{2}},
$$

for another constant $K^{\prime}$ and then the Hamiltonian which can be exactly integrated is

$$
H=\frac{p^{2}}{2}+\frac{1}{2} \frac{\omega_{0}^{2}}{\left(-K \omega_{0} t+K^{\prime}\right)^{2}} x^{2}
$$

and the corresponding Hamilton equations are

$$
\left\{\begin{array}{l}
\dot{x}=p \\
\dot{p}=-\frac{\omega_{0}^{2}}{\left(-K \omega_{0} t+K^{\prime}\right)^{2}} x
\end{array}\right.
$$

Had we used instead the family of curves in $S L(2, \mathbb{R})$ given by

$$
\bar{A}_{0}(t)=\left(\begin{array}{cc}
\frac{1}{V(t)} & 0 \\
-u_{1} & V(t)
\end{array}\right)
$$


where $u_{1}$ is a constant, and we would obtain the following relations among coefficients:

$$
\left\{\begin{aligned}
b_{2}^{\prime} & =V^{2} b_{2}+u_{1} V b_{1}+u_{1}^{2} b_{0}-u_{1} \dot{V} \\
b_{1}^{\prime} & =b_{1}+2 \frac{u_{1}}{V}-2 \frac{\dot{V}}{V} \\
b_{0}^{\prime} & =\frac{1}{V^{2}} b_{0}
\end{aligned}\right.
$$

Assume we want to relate the $t$-dependent vector field $X(\cdot, t)=X_{0}+F(t) \omega_{0}^{2} X_{2}$, characterised by $b_{0}=1, \quad b_{1}=0, \quad b_{2}=F(t) \omega_{0}^{2}$, with another one characterised by $b_{0}^{\prime}, b_{1}^{\prime}$ and $b_{2}^{\prime}$, which is integrable, or more explicitly, related with the $t$-dependent vector field $X(\cdot, t)=D(t)\left(c_{0} X_{0}+c_{2} X_{2}\right)$, i.e. $b_{0}^{\prime}=D c_{0}, b_{1}^{\prime}=0$, and $b_{2}^{\prime}=D c_{2}$,

Then $b_{1}=b_{1}^{\prime}=0$, and the second equation shows that $\dot{V}=u_{1}$, i.e. $V(t)=u_{1} t+u_{0}$ with $u_{0} \in \mathbb{R}$, and using this condition in the first equation, together with $b_{0}=1$, it becomes $b_{2}^{\prime}=V^{2} b_{2}$, and then, as the third equation gives us the value of $D$ as $c_{0} D=b_{0}^{\prime}=1 / V^{2}$, we see that $b_{2}^{\prime}=D c_{2}=V^{2} F(t) \omega_{0}^{2}$, and therefore $F$ must be proportional to $\left(u_{1} t+u_{0}\right)^{-4}$ :

$$
F(t)=\frac{k}{\left(u_{1} t+u_{0}\right)^{4}}, \quad k=\frac{c_{2}}{c_{0} \omega_{0}^{2}} .
$$

We can fix $c_{2}=\omega_{0}^{2}$ and $c_{0}=1$. Thus $F(t)=\left(u_{1} t+u_{0}\right)^{-4}$.

Finally, note that the time-dependent transformation $\bar{A}_{0}(t)$ which performs the reduction is

$$
\left\{\begin{aligned}
x^{\prime} & =\frac{x}{V(t)} \\
p^{\prime} & =-u_{1} x+V(t) p
\end{aligned}\right.
$$

which transforms the initial system of differential equations into

$$
\left\{\begin{aligned}
\frac{d x^{\prime}}{d t} & =\frac{1}{V^{2}(t)} p^{\prime} \\
\frac{d p^{\prime}}{d t} & =\frac{1}{V^{2}(t)}\left(-\omega_{0}^{2} x^{\prime}\right)
\end{aligned}\right.
$$

Now, using the time-reparametrization

$$
\tau(t)=\int_{0}^{t} \frac{d t^{\prime}}{V^{2}\left(t^{\prime}\right)}
$$

we obtain the following time-independent linear system

$$
\left\{\begin{array}{l}
\frac{d x^{\prime}}{d \tau}=p^{\prime} \\
\frac{d p^{\prime}}{d \tau}=-\omega_{0}^{2} x^{\prime}
\end{array}\right.
$$

whose general solution is

$$
\left(\begin{array}{c}
x^{\prime}(\tau) \\
p^{\prime}(\tau)
\end{array}\right)=\left(\begin{array}{cc}
\cos \left(\omega_{0} \tau\right) & \frac{\sin \left(\omega_{0} \tau\right)}{\omega_{0}} \\
-\omega_{0} \sin \left(\omega_{0} \tau\right) & \cos \left(\omega_{0} \tau\right)
\end{array}\right)\left(\begin{array}{c}
x^{\prime}(0) \\
p^{\prime}(0)
\end{array}\right)
$$


and thus we obtain that

$$
x(t)=V(t)\left(\cos \left(\omega_{0} \tau(t)\right) \frac{x_{0}}{V(0)}+\frac{1}{\omega_{0}} \sin \left(\omega_{0} \tau(t)\right)\left(-u_{1} x_{0}+V(0) p_{0}\right)\right)
$$

where we have used

$$
x^{\prime}(0)=\frac{x_{0}}{V(0)}, \quad p^{\prime}(0)=-u_{1} x_{0}+V(0) p_{0} .
$$

The same computations are valid for the Pinney equation when $F(t) \omega_{0}^{2}$ is replaced by a time-dependent angular frequency $\omega^{2}(t)$.

\section{Acknowledgments}

Partial financial support by research projects MTM2006-10531 and E24/1 (DGA) and a F.P.U. grant from Ministerio de Educación y Ciencia are acknowledged.

\section{References}

[1] J.F. Cariñena and A. Ramos, Int. J. Mod. Phys. A 14, (1999) 1935-51.

[2] S. Lie, Vorlesungen über continuierliche Gruppen mit Geometrischen und anderen Anwendungen, Edited and revised by G. Scheffers, Teubner, Leipzig, 1893.

[3] P. Winternitz, Lie groups and solutions of nonlinear differential equations, in: Nonlinear Phenomena, K.B. Wolf Ed., Lecture Notes in Physics 189, Springer-Verlag, N.Y., 1983

[4] J.F. Cariñena, J. Grabowski and G. Marmo, Lie-Scheffers systems: a geometric approach, Bibliopolis, Napoli, 2000.

[5] J.F. Cariñena, J. Grabowski and G. Marmo, Rep. Math. Phys. 60, (2007) 237-58

[6] R.L. Anderson, J. Harnad and P. Winternitz, Lett. in Math. Phys. 5, (1981) 143-148.

[7] J. Harnad, P. Winternitz and R.L. Anderson, J. Math. Phys. 24, (1983) 1062-72.

[8] S. Shnider and P. Winternitz, J. Math. Phys. 25, (1984) 3155-65.

[9] M.A. del Olmo, M.A. Rodríguez and P. Winternitz, J. Math. Phys. 27, (1986) 14-23

[10] M.A. del Olmo, M.A. Rodríguez and P. Winternitz, Integrability, chaos and nonlinear superposition formulas for differential matrix Riccati equations, in: Quantum Chaos and Statistical Nuclear Physics, pp. 372-378, Lecture Notes in Physics 263, 1986,

[11] M.A. del Olmo, M.A. Rodríguez and P. Winternitz, J. Math. Phys. 28, (1987) 530-535.

[12] J.F. Cariñena and A. Ramos, Acta Appl. Math. 70, (2002) 43-69.

[13] J.F. Cariñena, A. Ramos and J. de Lucas, Elect. J. Diff. Eqs. 122, (2007) 1-14.

[14] W.E. Milne, Phys. Rev. 35, (1930) 863-67.

[15] E. Pinney, Proc. A.M.S. 1, (1950) 681.

[16] J. F. Cariñena and A. Ramos, Lie systems and Connections in fibre bundles: Applications in Quantum Mechanics, in: $9^{\text {th }}$ Int. Conf. Diff. Geom and Appl., p. 437-452 (2004), J. Bures et al. Eds., (Matfyzpress, Praga, 2005).

[17] P. Caldirola, Nuovo Cim. 18, (1941) 393-400.

[18] E. Kanai, Prog. Theor. Phys. 3, (1948) 440-42.

[19] J. Wei and E. Norman, J. Math. Phys. 4, (1963) 575-81.

[20] J.F. Cariñena, J. Grabowski and A. Ramos, Acta Appl. Math. 66, (2001) 67-87.

[21] J.F. Cariñena, J. de Lucas and M.F. Rañada, Nonlinear superpositions and Ermakov systems, in: Differential Geometric Methods in Mechanics and Field Theory, pp. 15-33, eds F. Cantrijn, M. Crampin and B. Langerock (Academia Press, 2007)

[22] V. M. Strelchenya, J. Phys. A: Math. Gen. 24, (1991) 4965-4967. 